\title{
Relevant Issues in Forensic Biomechanics
}

John J Smith*

President Raymond P Smith and Associates, Denver, Colorado, USA

"Corresponding author: John J Smith, PE, MSBMT, MSEE, President Raymond P Smith and Associates, Denver, Colorado, USA, Tel: 303-840-0549; Fax: 303-805-5810; E- mail: JJSakaJTM@aol.com

Received date: November 08, 2012; Accepted date: December 17, 2012; Published date: December 19, 2012

Copyright: $@ 2014$ Smith JJ. This is an open-access article distributed under the terms of the Creative Commons Attribution License, which permits unrestricted use, distribution, and reproduction in any medium, provided the original author and source are credited.

\section{Editorial}

\section{John Smith,}

One aspect of forensic biomechanics is the merger of what occurs in the real world with what is can be measured, analyzed and established in a laboratory. To the casual observer, the difference between the data in these sources would seem to be insurmountable. Test data shows volunteer subjects experiencing changes in velocity without injury at levels significantly greater than those where people are injured in collisions on the roadway. Injuries are well documented in actual collisions that do not appear to match the data from tests. Crash tests reveal extensive vehicle damage in one type of collision and no damage at higher speeds in a different type of collision. However, when a proper analysis is performed, the discrepancies in the sources should be resolvable.

The limitations of research in laboratories are well known but include restrictions on test subjects and boundaries on test conditions. With regard to the former, it is proper that living human subjects cannot be tested to failure. With regard to the latter, when biomechanically relevant variables are considered the test conditions can address very few of the more than one trillion permutations that can occur in a traumatic event. However, the limitations of data collected from real collisions on the roadway often include reliance on secondary sources and incomplete information, just to name a few.

If it is accepted that a goal in forensic biomechanics is to apply the principles of engineering and science to understanding what occurs in given events, then this raises the question of what can be done to reconcile the sources of information. It also generates a series of questions that can provide possibilities for areas of research and analysis.

There exist numerous areas of disagreement in the forensic biomechanics community regarding motor vehicle collisions. The following list of potential research areas does not encompass all of them but does provided a starting point. The following questions should be addressed by the forensic biomechanics community.

Is there an identified threshold for injury for the motoring public? DOT provides performance standards, not injury thresholds. Laboratory tests protect the subjects from injury, as is required. If there is a threshold, what is the proper metric? Change in velocity is easy to use but effectively requires rigid body analysis which is not biomechanically valid. Is acceleration relevant without considering differential movement?

What are the limits in extrapolating volunteer data to actual collisions? In what areas is the use of the data appropriate? As an example, COL Stapp tested volunteers on rocket sleds. How much of that data is applicable to actual motor vehicle collisions?

Can a motor vehicle collision be reasonably compared to a different activity? An argument is often made that a traumatic event can be compared to a non-traumatic daily activity. How important are the issues of learned response, muscle activation times, source of applied forces, awareness, differential body movement etc.?

How relevant is the damage to the vehicle in determining the injuries to the occupant? Is damage a reliable indicator of the type and severity of the injuries? It is established that certain injury patterns can be correlated with the type and location of applied forces. How much does vehicle damage play into this?

How can damage in different types of collisions be correlated? Applying the principles of conservation of energy, it is not surprising there is more damage in a barrier impact when compared to a vehicle to vehicle impact at the same speed. Rather than discard thousands of barrier impacts, how can the data be properly factored into an analysis?

What are the biomechanically relevant factors that mitigate or aggravate injury potential? As an example, it is well established that women are injured at lower levels and higher rates than men. While there are many theories for this variation, it does demonstrate one aspect of an area for further research.

What are the limits of computer modeling? Finite element modeling has come a long way but cannot capture all of the key variables.

While the list above is not all inclusive, it does provided a starting point for researchers attempting to bridge the gaps in forensic knowledge. While I have opinions on all of them, it would be beneficial for the forensic biomechanical community to address these issues in an open and professional manner. 